\title{
A mini review on neurofibromatosis type 1 from the radiological point of view
}

Monika Bekiesinska-Figatowska ${ }^{1 *}$

'Department of Diagnostic Imaging, Institute of Mother and Child, Kasprzaka 17a, 01-211 Warsaw, Poland

Article Info

\section{Article Notes}

Received: October 29, 2017

Accepted: December 27, 2017

\section{*Correspondence:}

Prof. Monika Bekiesinska-Figatowska, Department of Diagnostic Imaging, Institute of Mother and Child,

Kasprzaka 17a,01-211 Warsaw, Poland;

Telephone: +48 22 3277156;

Fax: +48 223277 195;

Email: m.figatowska@gmail.com

C 2017 Bekiesińska-Figatowska M. This article is distributed under the terms of the Creative Commons Attribution 4.0 International License.

\section{Keywords}

neurofibromatosis type 1 (NF1)

manifestations

diagnostic imaging

diagnostics

follow-up

\section{ABSTRACT}

Neurofibromatosis type one (NF1) belongs to the most frequent rare diseases, requiring various methods of diagnostic imaging at different stages of diagnostics and follow-up. Magnetic resonance imaging (MRI) is a method of choice in diagnostics of brain: unidentified neurofibromatosis objects, optic pathway gliomas and other tumours which can spontaneously regress. NF1 patients suffer from vascular abnormalities with a predominance of aortic, renal, mesenteric, and carotid-vertebral stenoses or aneurysms. These are evaluated by ultrasound, computed tomography- or MRI-angiography and by digital subtraction angiography. In our material we described twofold higher occurrence of arterial variants in brain in NF1 than in the control group. Characteristic skeletal changes include tibial pseudarthrosis, scoliosis, sphenoid wing dysplasia, rib penciling, and gracile bones, usually diagnosed with plain radiographs. Outside CNS we deal with neurofibromas and plexiform neurofibromas in any location in the body. Their malignant transformation leads to development of malignant peripheral nerve sheath tumour, or malignant triton tumour. Rhabdomyosarcoma, juvenile myelomonocytic leukemia and phaeochromocytoma are also encountered in NF1 patients with increased frequency. Regular imaging follow-up studies should not be routinely performed in NF1 patients. MRI is recommended for follow-up of clinically suspected tumours, single whole-body MR is recommended at transition to adulthood. Positron emission tomography/computed tomography is useful for the detection of malignant transformation of tumours in NF1 patients.

Neurofibromatosis type one (NF1) belongs to the most frequent rare diseases, occurring with an incidence of approximately one in 3000 individuals at birth ${ }^{1}$. It has cutaneous, ocular, neurologic, musculoskeletal, vascular and cardiac manifestations, including tumours. Various methods of diagnostic imaging are used at different stages of diagnostics and follow-up of many of these manifestations. Institute of Mother and Child in Warsaw, Poland, is a tertiary referral centre for NF1 patients and diagnostic imaging belongs to the routine work-up of these patients ${ }^{2}$.

The diagnostic criteria for NF1 were defined in 1997 by the National Institute of Health (NIH) Criteria Consensus Conference and are as follows:

- six or more spots of "cafe au lait" type more than $5 \mathrm{~mm}$ in diameter,

- two or more neurofibromas or one plexiform neurofibroma,

- freckles or skin discolorations in the places inaccessible for light (armpits, groins, pubic region), 
- two or more Lisch nodules on the iris

- characteristic skeletal changes,

- a first-degree relative suffering from $\mathrm{NF}^{3}$.

At least two criteria must be met for clinical diagnosis.

Skin lesions such as café-au-lait spots or freckling on flexural areas, and Lisch nodules will not be a subject of these considerations as they do not require diagnostic imaging.

Radiological features of NF1 in central nervous system are well-known and widely described in the literature and have been in the field of interest of our centre as well as other manifestations of the disease ${ }^{2,4}$. Unidentified neurofibromatosis objects (UNO), formerly called unidentified bright objects (UBO) detected on magnetic resonance imaging (MRI) are typical of NF1. Basal ganglia are the most frequent localisation of these lesions ${ }^{5}$ as well as cerebellum and brainstem ${ }^{6}$. UNO show hyperintense signal on T2-weighted images (T2WI), including T2FLAIR, and are usually isointense on T1-weighted images (T1WI), but lesions located in the globi pallidi often display hyperintense signal also on $\mathrm{T}^{\mathrm{W}} \mathrm{WI}^{5}$. UNO can also be found in the corpus callosum, especially in the callosal splenium, but in our experience this finding is less frequent ${ }^{7}$.

There is some degree of difficulty when assessing the thalami and medial parts of the temporal lobes (hippocampi, parahippocampal structures, amygdala, sometimes with extension into the insula) which are thickened and slightly diffusely hyperintense on T2WI in NF1 patients (figure. 1). This observation has already been mentioned in the literature. Gill et al. report possible different pathological basis of temporal lesions which do not resolve with age as opposed to lesions in the basal ganglia, thalami, cerebellum and brainstem that do resolve ${ }^{6}$.

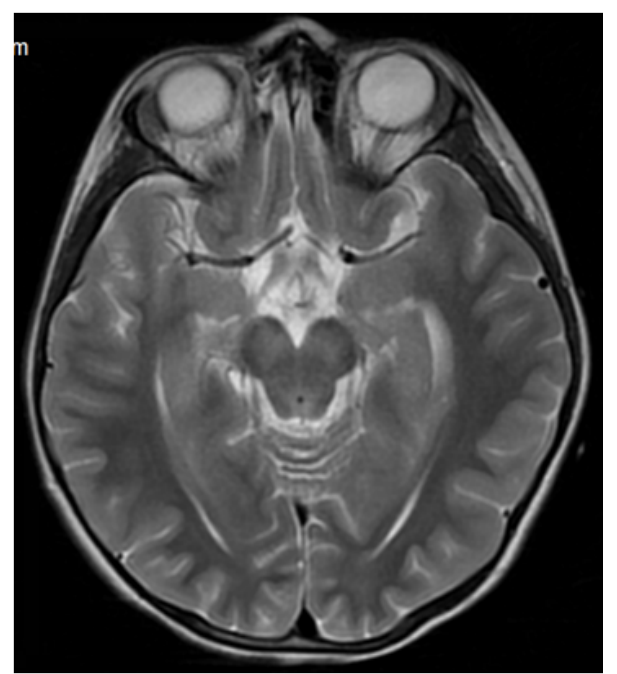

Figure 1. MRI, FSE/T2WI, axial plane. Slightly thickened and discretely hyperintense medial parts of temporal lobes in a 9-yearold girl with NF1.

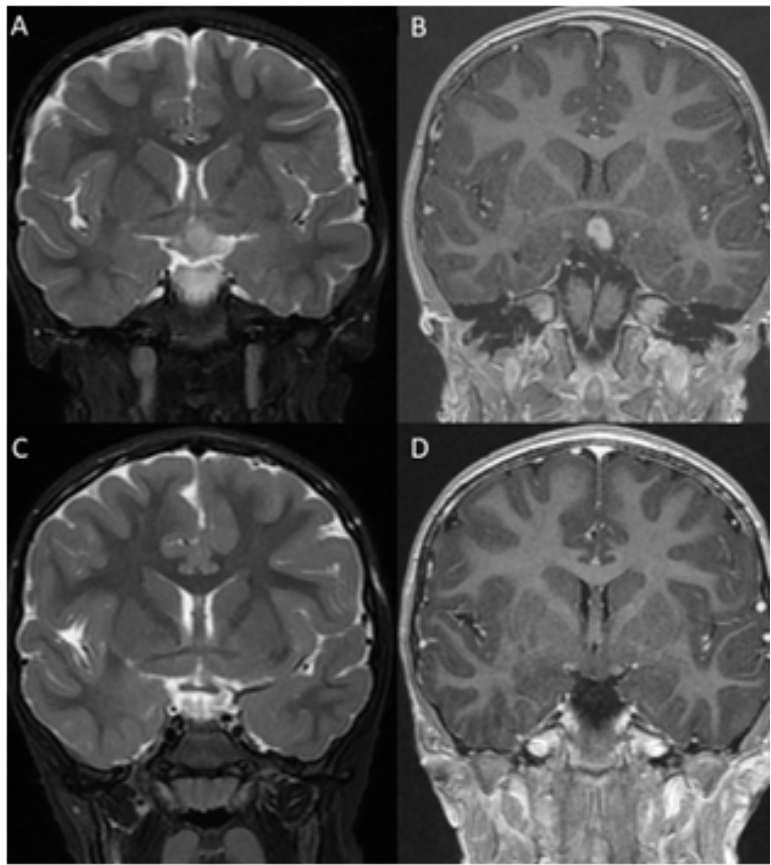

Figure 2. MRI, coronal plane, A, C - FSE/T2WI, B, D - FSPGR/3D/ T1WI post-gadolinium. A, B. Four-year-old girl with NF1 and an enhancing hypothalamic glioma. C, D. One year later - complete regression of the tumour without treatment.

Pilocytic astrocytoma, forming optic pathway glioma (OPG), constitutes a major management issue. Until 8 years of age, clinical assessment for this pathology is recommended every 6 to 12 months, but routine MRI assessment is not advised in asymptomatic patients with NF1 and no clinical signs of visual pathway disturbances ${ }^{8}$. Treatment for symptomatic OPG (with strabismus, exophthalmos, amblyopia, endrocrinological disturbances) is not well standardized ${ }^{9}$. Various lines of chemotherapy are used with minimal effect on the solid components of the tumours initially but no effect on cystic ones and with subsequent growth of the tumour regardless of treatment ${ }^{10}$. Clinical findings do not differ between treated patients and those who are subjected to the "watch and wait" policy ${ }^{9}$. Besides, spontaneous regression of optic pathway and/or hypothalamic gliomas in NF1 patients has been described (figure 2), there are also reported cases of spontaneous resolution of non-optical gliomas in the literature ${ }^{11}$.

There is another difficulty in the assessment of mass lesions in the brainstem of NF1 patients. Diffuse, often asymmetrical pontine and/or medullary enlargement with frequently very discrete hyperintensity on T2WI may also spontaneously regress over time thus indicating UNO rather than brain stem glioma ${ }^{12}$. MR spectroscopy (MRS) is helpful in these situations with a high choline to creatine ratio over 2 (Cho/Cr $>2$ ) typical of a tumour ${ }^{13}$.

NF1 patients suffer from vascular abnormalities with a predominance of aortic, renal, mesenteric, and carotid- 
vertebral stenoses or aneurysms in the younger patients, and degenerative atherosclerotic aortic aneurysms in the older ones ${ }^{14}$. Renal artery stenosis caused by NF1 vasculopathy is a well-known cause of hypertension ${ }^{15}$. Ultrasonography (US), CT- and/or MR-angiography and in some cases digital subtraction angiography (DSA) are employed in diagnostic process of vascular manifestations of NF1. Cerebral arteriopathy which is reported to be more prevalent in NF1 patients than in general population has not been found in our experience (except one case), at least that with clinical significance. We have described twofold higher occurrence of arterial variants in NF1 patients than in the control group, the most frequent variant being fetal configuration of the circle of Willis (defined as P1 part of the posterior cerebral artery smaller than posterior communicating artery with ipsilateral P2 supplied by internal carotid artery via posterior communicating artery). Multiple variants were more frequent in NF1 patients than in healthy controls although this difference did not reach statistical significance. The only NF1 patient with significant vascular condition in our material had moyamoya disease ${ }^{16}$. However, followed-up for 6 years now, he is still free from stroke and its neurological consequences.

Characteristic skeletal changes of NF1 include congenital pseudarthrosis of the tibia, scoliosis, sphenoid wing dysplasia, rib penciling, and gracile bones ${ }^{17}$. Usually plain radiographs are sufficient to diagnose these manifestations of the disease and computed tomography (CT) is used for special indications ${ }^{18,19}$. NF1 predisposes patients to benign and malignant tumour development, however, bone malignancies are extremely rare in this population ${ }^{20}$. Outside CNS we deal with neurofibromas and plexiform neurofibromas in any location in the body of NF1 patients. The routine screening method is US. We use MRI when we are looking for compression of vital structures, e.g. of the spinal cord by the tumours extending from the intervertebral foramina or of the trachea, and for infiltration of other organs when considering resectability of the tumour (figure 3) and - first of all - for the signs of malignancy in rapidly growing tumours (figure 4). Malignant transformation leads to the development of such tumours as malignant peripheral nerve sheath tumour (MPNST), or malignant triton tumour. Other soft tissue sarcomas, e.g. rhabdomyosarcoma (RMS), as well as other malignancies such as juvenile myelomonocytic leukemia or phaeochromocytoma are also encountered in NF1 patients with increased frequency comparing to general population 8,15. However, for example the Endocrine Society, European Society of Endocrinology, and American Association for Clinical Chemistry do not recommend screening asymptomatic NF1 patients for pheochromocytoma and paraganglioma ${ }^{21}$.

It is no more questionable whether regular imaging follow-up studies, often required by the clinicians, should be routinely performed in NF1 patients ${ }^{8}$. It has been stated in the literature at least 15 years ago by French

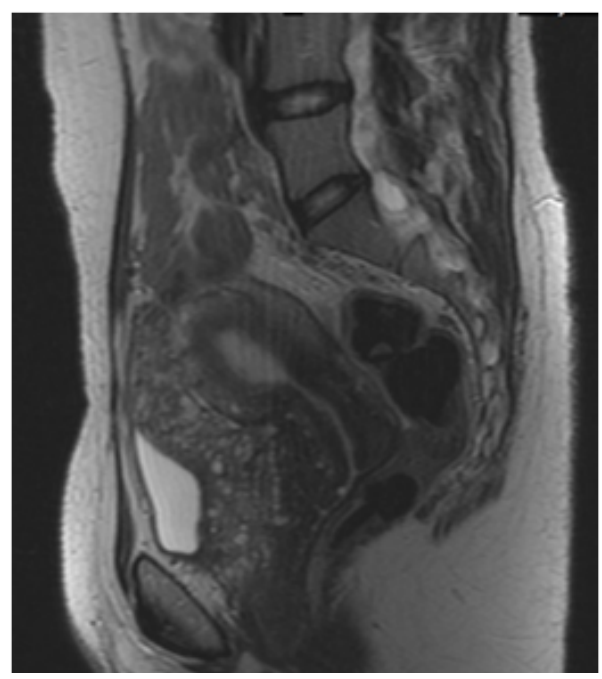

Figure 3. MRI, FSE/T2WI, sagittal plane. Female NF1 patient at the age of 26 years. Plexiform neurofibroma of the pelvis infiltrating anterior wall of the uterus and posterior wall of the bladder. The attempted tumor resection was unsuccessful, the surgeons were unable to remove the whole lesion.

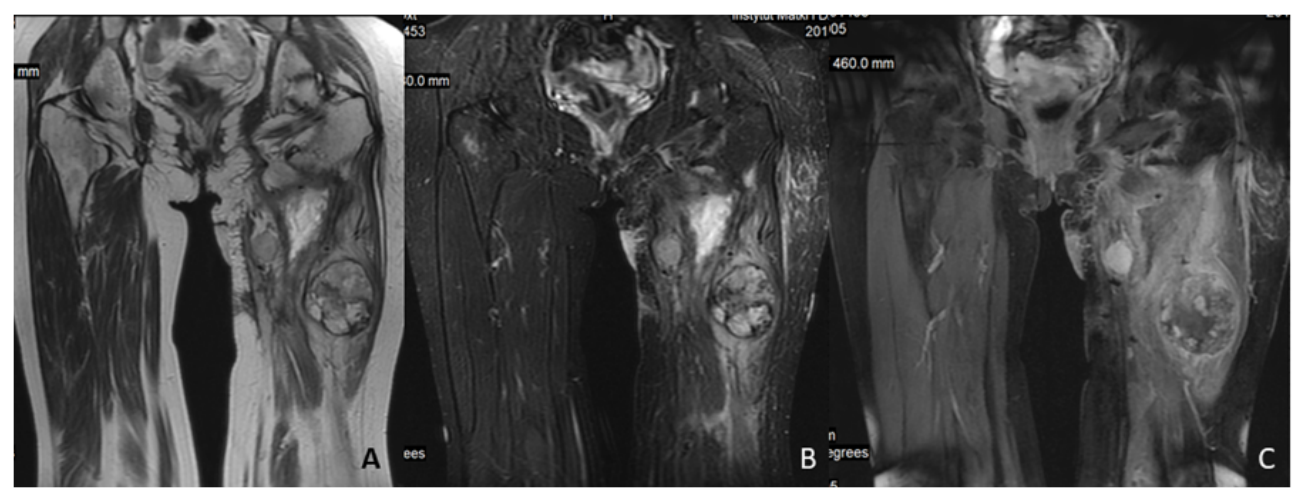

Figure 4. MRI, coronal planes, $\mathrm{A}-\mathrm{FSE} / \mathrm{T} 2 \mathrm{WI}, \mathrm{B}-\mathrm{STIR}, \mathrm{C}-\mathrm{FSE} /$ fat-saturated T1WI post-gadolinium. Recurrence of MPNST in the left thigh in a 26-year old woman with NF1. 
experts ${ }^{22}$. It has also been stated earlier, in 1992, by the participants of a joint World Health Organization and National Neurofibromatosis Foundation meeting that "although some clinicians have advocated routine screening scans for all patients with NF1, the utility of such screening has not been conclusively demonstrated" ${ }^{23}$. Also the experience of our centre does not show real advantage of this approach. After years of gaining experience, our way of thinking has changed and we now do not share the point of view of oncologists/oncological surgeons published years ago that " 'watchful waiting' with regular MR monitoring ... if there are no oncological reasons for anxiety" should be standard management of NF1 patients ${ }^{2,24}$. It is more reasonable to perform follow-up MRI in cases of new/progressing clinical signs and symptoms. Repeated sedation (in paediatric patients, as in our case) and gadolinium-based contrast media administration should be weighed against real clinical benefit from this kind of management. The finding of gadolinium deposition in the brain despite normal renal function, that has been a concern so far in terms of nephrogenic systemic fibrosis 25 , has pointed at its possible long-term toxicity. We know that more stable macrocyclic contrast agents should be the primary choice but avoiding unnecessary use is the key point ${ }^{26}$. In the literature, annual clinical examination is recommended except in cases with complications. Taking into account the rarity of complications that are usually symptomatic and easily detected during the clinical followup, screening investigations are not recommended ${ }^{8,22}$. The only controversial exception was MRI for early detection of optic pathway gliomas in children ${ }^{22}$ but it is not advised any more now ${ }^{8}$. At present MRI is generally recommended for follow-up of clinically suspected tumours, both intra- and extracranial ${ }^{27}$ and it is recommended to consider a single whole-body MR examination at transition to adulthood which might assist in determining approaches to long-term follow-up in the future life of a patient ${ }^{8}$. Positron emission tomography/computed tomography (PET/CT) is useful for

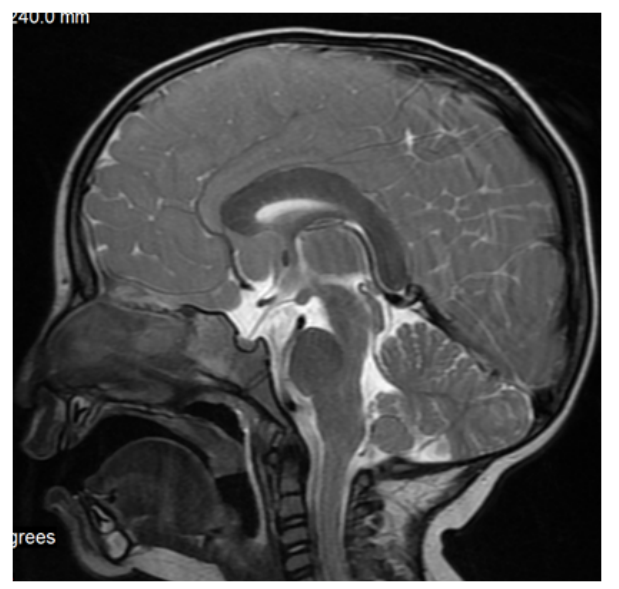

Figure 5. MRI, FSE/T2WI, sagittal plane. Significantly increased thickness of the corpus callosum an a 2-year-old boy with NF1. the detection of malignant transformation of tumours in NF1 patients ${ }^{28}$.

Despite our wide knowledge concerning NF1 there are still numerous issues to be addressed. Learning disabilities, attention deficits, and social and behavioural problems including autism spectrum disorder, decrease the quality of life of NF1 patients. Advanced MRI techniques, like Diffusion Tensor Imaging (DTI) or functional MRI (fMRI), are more and more widely used to explore these domains ${ }^{29,30}$. For example, the relationship between increased brain volume, including the volume of the corpus callosum, and decreased IQ in NF1 patients still remains to be explained (figure 5). This is the purpose of our ongoing research with use of MRvolumetry.

\section{Conflict of interest}

The author declares no conflict of interest.

The author received no funding.

\section{References}

1. Evans DG, Howard E, Giblin C, et al. Birth incidence and prevalence of tumor-prone syndromes: estimates from a UK family genetic register service. Am J Med Genet A. 2010; 152A: 327-332.

2. Jastrzebska M, Bekiesinska-Figatowska M, Karwacki M, et al. MR imaging features in children with type 1 neurofibromatosis (NF1): Intra- and extracranial lesions. Pol J Radiol. 2009; 74(2): 14-21.

3. Neurofibromatosis: Conference statement. National Institute of Health Consensus Development Conference (1988). Arch Neurol 1988; 45: 575-578.

4. Jacques C, Dietemann JL. [Imaging features of neurofibromatosis type 1]. J Neuroradiol. 2005; 32(3): 180-197.

5. Bekiesinska-Figatowska M, Mierzewska H, Jurkiewicz E. Basal ganglia lesions in children and adults. Eur J Radiol. 2013; 82: 837-849.

6. Gill DS, Hyman SL, Steinberg A, et al. Age-related findings on MRI in neurofibromatosis type 1. Pediatr Radiol. 2006; 36(10): 1048-1056.

7. Krupa K, Bekiesinska-Figatowska M. Congenital and Acquired Abnormalities of the CorpusCallosum: A Pictorial Essay. Biomed Res Int. 2013, Article ID 265619, 14 pages, 2013. doi:10.1155/2013/265619.

8. Evans DGR, Salvador H, Chang VY, et al. Cancer and Central Nervous System Tumor Surveillance in Pediatric Neurofibromatosis 1. Clin Cancer Res. 2017 Jun 15; 23(12): e46-e53.

9. Friedrich RE, Nuding MA. Optic Pathway Glioma and Cerebral Focal Abnormal Signal Intensity in Patients with Neurofibromatosis Type 1: Characteristics, Treatment Choices and Follow-up in 134 Affected Individuals and a Brief Review of the Literature. Anticancer Res. 2016; 36(8): 4095-4121.

10. Shofty B, Mauda-Havakuk M, Weizman L, et al. Pediatr Blood Cancer. 2015; 62(8): 1353-1359.

11. Cakirer S, Karaarslan E. Spontaneous involution of a non-optic astrocytoma in neurofibromatosis type I: serial magnetic resonance imaging evaluation. Acta Radiol. 2004; 45(6): 669-673.

12. Hervey-Jumper SL, Singla N, Gebarski SS, et al. Diffuse pontine lesions in children with neurofibromatosis type 1 : making a case for unidentified bright objects. Pediatr Neurosurg. 2013; 49(1): 55-59.

13. Gonen $\mathrm{O}$, Wang ZJ, Viswanathan AK, et al. Three-dimensional multivoxel proton MR spectroscopy of the brain in children with neurofibromatosis type 1. Am J Neuroradiol. 1999; 20(7): 1333-1341. 
14. Oderich GS, Sullivan TM, Bower TC, et al. Vascular abnormalities in patients with neurofibromatosis syndrome type I: clinical spectrum, management, and results. J Vasc Surg. 2007; 46(3): 475-484.

15. Friedman JM, Arbiser J, Epstein JA, et al. Cardiovascular disease in neurofibromatosis 1: report of the NF1 Cardiovascular Task Force. Genet Med. 2002; 4(3): 105-111.

16. Bekiesińska-Figatowska M, Brągoszewska H, Duczkowski M, et al. Circle of Willis abnormalities in children with neurofibromatosis type 1. Neurol Neurochir Pol. 2014; 48(1): 15-20.

17. Schindeler A, Little DG. Recent insights into bone development homeostasis, and repair in type 1 neurofibromatosis (NF1). Bone. 2008; 42(4): 616-622.

18. Alwan S, Armstrong L, Joe $\mathrm{H}$, et al. Associations of osseous abnormalities in Neurofibromatosis 1. Am J Med Genet A. 2007; 143A(12): 1326-1333.

19. Li Y, Luo M, Wang W, et al. A Computed Tomography-Based Comparison of Abnormal Vertebrae Pedicles Between Dystrophic and Nondystrophic Scoliosis in Neurofibromatosis Type 1. World Neurosurg. 2017; 106: 898-904.

20. Afşar CU, Kara IO, Kozat BK, et al. Neurofibromatosis type 1, gastrointestinal stromal tumor, leiomyosarcoma and osteosarcoma: four cases of rare tumors and a review of the literature. Crit Rev Oncol Hematol. 2013; 86(2): 191-199.

21. Lenders JWM, Duh QY, Eisenhofer G, et al. Endocrine Society. Pheochromocytoma and paraganglioma: an endocrine society clinical practice guideline J Clin Endocrinol Metab. 2014; 99(6): 1915-42.
22. Pinson S, Créange A, Barbarot S, et al. [Neurofibromatosis 1: recommendations for management]. Arch Pediatr. 2002; 9(1): 49-60.

23. Prevention and control of neurofibromatosis: memorandum from a joint WHO/NNFF meeting. Bull World Health Organ 1992; 70(2): 173-182.

24. Wozniak W, Karwacki MW. Is "watchful waiting" superior to surgery in children with neurofibromatosis type 1 presenting with extracranial and extramedullary tumor mass at diagnosis. Childs Nerv Syst. 2008; 24(12): 1431-1436.

25. Beam AS, Moore KG, Gillis SN, et al. GBCAs and Risk for Nephrogenic Systemic Fibrosis: A Literature Review. Radiol Technol. 2017; 88(6): 583-589.

26. Soares BP, Lequin MH, Huisman TAGM. Safety of Contrast Material Use in Children. Magn Reson Imaging Clin N Am. 2017; 25(4): 779-785.

27. Jett K, Friedman JM. Clinical and genetic aspects of neurofibromatosis 1. Genet Med. 2010; 12(1): 1-11.

28. Moharir M, London K, Howman-Giles R, et al. Utility of positron emission tomography for tumour surveillance in children with neurofibromatosis type 1. Eur J Nucl Med Mol Imaging. 2010; 37(7): 1309-1317.

29. Yoncheva YN, Hardy KK, Lurie DJ, et al. Computerized cognitive training for children with neurofibromatosis type 1: A pilot restingstate fMRI study. Psychiatry Res. 2017; 266: 53-58.

30. Koini M, Rombouts SA, Veer IM, et al. White matter microstructure of patients with neurofibromatosis type 1 and its relation to inhibitory control. Brain Imaging Behav. 2016 Oct 29. [Epub ahead of print] 